\title{
Deficiência de zinco em crianças e adolescentes com doenças hepáticas crônicas
}

\author{
Zinc deficiency in children and adolescents with chronic liver diseases
}

Thalita Cremonesi Pereira ${ }^{1}$, Gabriel Hessel ${ }^{2}$

\section{RESUMO}

Objetivo: Revisar as principais pesquisas referentes ao zinco e ao estado desse mineral em crianças e adolescentes com doenças hepáticas crônicas.

Fontes de dados: As palavras-chave "zinco", "hepatopatias", "criança" e "adolescente" foram inseridas nas bases de dados PubMed, SciELO e Web of Science. O critério de seleção compreendeu os artigos de origem nacional e internacional, preferindo-se aqueles publicados de 1998 a 2008, além de estudos mais antigos considerados clássicos.

Síntese dos dados: O zinco é um mineral essencial para a saúde das crianças devido às suas inúmeras funções no organismo, dentre elas a atuação no sistema imune, o favorecimento do crescimento estatural e do desenvolvimento sexual e cognitivo. As crianças hepatopatas parecem estar mais suscetíveis à deficiência de zinco do que as saudáveis pelo fato de a doença no fígado alterar o metabolismo desse mineral, principalmente a sua distribuição aos tecidos e sua excreção. O nível de zinco no plasma parece ser baixo nesses pacientes, mas esse biomarcador não reflete o real estado de zinco no organismo e, além disso, a excreção urinária de zinco parece estar aumentada.

Conclusões: É necessário um número maior de estudos sobre o estado de zinco em crianças e adolescentes com doenças hepáticas crônicas.

Palavras-chave: zinco; hepatopatias; criança; adolescente.

\section{ABSTRACT}

Objective: To review the literature related to zinc and zinc level in children and adolescents with chronic liver diseases.

Data sources: The following key-words were used to retrieve studies from PubMed, SciELO and Web of Science databases: "zinc", "liver diseases", "child" and "adolescent". National and international studies published from 1998-2008 were selected as well as classical studies on the theme.

Data synthesis: Zinc is an essential mineral for children's health with several functions in the organism, improving defense mechanisms, growth, sexual and cognitive development. Children with liver diseases seem to be more susceptible to zinc deficiency than healthy children, since their liver illness modifies the metabolism of this mineral, mainly regarding the distribution on tissues and elimination. The zinc level in the plasma seems to be low in these patients, but this index does not reflect the real zinc status in the organism; additionally, the urinary elimination of zinc seems to increase.

Conclusions: More studies are needed in order to understand zinc status in children and adolescents with chronic liver diseases.

Keywords: zinc; liver diseases; child; adolescent.
Instituição: Faculdade de Ciências Médicas da Universidade Estadual de Campinas (FCM-Unicamp), Campinas, SP, Brasil

${ }^{1}$ Nutricionista; aluna de Mestrado do curso Saúde da Criança e do Adolescente da FCM-Unicamp, Campinas, SP, Brasil

${ }^{2}$ Professor associado do Departamento de Pediatria da FCM-Unicamp, Campinas, SP, Brasil
Fontes financiadoras: Coordenação de Aperfeiçoamento de Pessoal de Nível Superior (Capes) e Fundação de Amparo à Pesquisa do Estado de São Paulo (Fapesp).

Recebido em: 8/7/08 Aprovado em: 27/10/08 


\section{Introdução}

Tem sido relatada a importância do zinco ( $\mathrm{Zn}$ ) no organismo dos seres humanos, principalmente crianças, as quais são mais suscetíveis à deficiência de Zn, que pode levar a inúmeros prejuízos em seu desenvolvimento ${ }^{(1,2)}$.

Esta carência parece afetar com maior gravidade pessoas com algum tipo de enfermidade ${ }^{(3,4)}$ e pode ser mais prevalente nos pacientes com doenças hepáticas crônicas pelo fato de o fígado ser o órgão responsável pela distribuição de nutrientes aos demais tecidos, o que deixaria tais pacientes mais expostos à deficiência de $\mathrm{Zn}^{(2,5)}$. O objetivo desta pesquisa foi revisar os principais estudos referentes ao $\mathrm{Zn}$ e verificar a frequência da deficiência de Zn nas crianças e adolescentes com doenças hepáticas crônicas. Para isso, as palavras-chave "zinco", "hepatopatias", "criança" e "adolescente" foram inseridas nas bases de dados PubMed, SciELO e Web of Science. O critério de seleção desses artigos compreendeu aqueles de origem nacional e internacional, dandose preferência àqueles publicados nos últimos dez anos.

\section{Funções do Zn}

O Zn é um mineral essencial que atua em diversas funções do organismo pelo fato de ser cofator de mais de 300 enzimas e proteínas, como a anidrase carbônica, a fosfatase alcalina, as carboxipeptidases, a álcool desidrogenase, a superóxido dismutase, a proteína $C$ quinase, a ácido ribonucleico polimerase e a transcriptase reversa ${ }^{(2,6)}$. Por isso, o $\mathrm{Zn}$ é importante em atividades do sistema imune, prevenção de formação de radicais livres, crescimento estatural, desenvolvimento sexual e cognitivo e síntese de DNA ${ }^{(2,5-7)}$.

\section{Recomendações nutricionais e alimentos com maior quantidade de $\mathrm{Zn}$}

O Zn é obtido apenas por meio da alimentação, sendo encontrado em grandes quantidades nos produtos de origem animal e nos frutos do mar, principalmente nas carnes vermelhas, mariscos, ostras, fígado, miúdos e ovos ${ }^{(2)}$. A recomendação de consumo de $Z n$ para indivíduos saudáveis varia conforme a idade. Para lactentes, a recomendação é de 2 a $3 \mathrm{mg} / \mathrm{dia}$, para crianças e adolescentes entre um e 18 anos varia de 3 a $11 \mathrm{mg} /$ dia, para adultos e idosos, esta recomendação está entre 8 e $11 \mathrm{mg} /$ dia e, para mulheres gestantes ou lactantes, o consumo ideal de Zn está entre 11 e $14 \mathrm{mg} / \mathrm{dia}^{(8)}$.

O Institute of Medicine, órgão responsável pelas recomendações de nutrientes nos EUA e Canadá, também estabeleceu o limite máximo tolerável (UL) para a ingestão de Zn em indivíduos saudáveis a fim de evitar sinais clínicos do seu excesso no organismo, variando conforme a idade: 7 a $34 \mathrm{mg} /$ dia para crianças e adolescentes, podendo chegar ao valor de $40 \mathrm{mg} /$ dia para adultos ${ }^{(8)}$.

\section{Biodisponibilidade e metabolismo de Zn}

É importante considerar que nem toda quantidade de Zn ingerida pela alimentação é utilizada pelo organismo, pois sua biodisponibilidade pode ser afetada no processo de absorção intestinal ou já na circulação sanguínea. A absorção intestinal de $\mathrm{Zn}$ é diminuída por fatores antagonistas na alimentação, como o fitato, o oxalato, os taninos e os polifenóis. Tal absorção pode ser facilitada pela presença de aminoácidos (cisteína e histidina), fosfatos, ácidos orgânicos e proteína ${ }^{(5,9,10)}$. Na circulação, pode haver competição do $\mathrm{Zn}$ com os minerais cobre e ferro, dependendo da quantidade desses elementos na corrente sanguínea ${ }^{(6)}$.

A maior absorção de Zn parece ocorrer no jejuno, e a captação desse nutriente é regulada pela difusão ou por carreadores, sendo o último mais utilizado quando há baixa quantidade de Zn. Após absorção e captação, o mineral é transportado no sangue portal e o fígado tem a função de distribuí-lo aos demais tecidos ${ }^{(2,11)}$.

$\mathrm{O} \mathrm{Zn}$ encontrado no organismo de adultos corresponde a cerca de $2 \mathrm{~g}$. Dessa quantidade, $80 \%$ está presente nos músculos e $\operatorname{ossos}^{(5)}$. Na corrente sanguínea, o Zn encontra-se nos eritrócitos (90\%), plasma (9\%) e leucócitos (menos que 1\%). Apesar de haver pouco Zn nos glóbulos brancos do sangue, a sua quantidade, quando calculada por número de células, é mais concentrada, sendo cerca de sete vezes maior do que nas células vermelhas ${ }^{(12)}$. O Zn encontrado no plasma está em sua maior parte ligado à albumina $(80 \%)$ e os $20 \%$ restantes estão ligados à alfa-2-macroglobulina ${ }^{(13)}$.

A eliminação do $Z n$ do organismo é realizada por meio da urina e das fezes: cerca de $0,5 \mathrm{mg}$ de $\mathrm{Zn}$ é eliminado pela urina e $2 \mathrm{mg}$ pelas fezes por dia. Contudo, quando se detecta ausência ou pequenas quantidades de $\mathrm{Zn}$ na alimentação, o organismo tenta conservar valores normais de $\mathrm{Zn}$ nos tecidos e no sangue para evitar a deficiência desse mineral, processo denominado mecanismo homeostático. A forma como o organismo consegue realizar o mecanismo homeostático ainda permanece sem explicações definidas, mas há evidências de que se relaciona com o processo de absorção e excreção de $\mathrm{Zn}^{(14-16)}$. 


\section{Deficiência de Zn}

O primeiro relato de deficiência de $\mathrm{Zn}$ veio do Egito, em 1963 , e reporta que homens sofriam de retardo do crescimento e prejuízo da maturação sexual (hipogonadismo) consequentes à deficiência do mineral. A ocorrência dessa deficiência, segundo os autores, provavelmente se originou de uma dieta rica em fitato, o qual pode ter inibido a absorção de $\mathrm{Zn}^{(17)}$.

Os sinais e sintomas clínicos da deficiência de Zn incluem, além dos dois fatores já citados, diminuição do apetite e paladar (hipogeusia), diminuição das funções cognitivas, acrodermatite enteropática (alopecia, diarreia e lesões na pele) e deficiências no sistema imune ${ }^{(6,7,18,19)}$.

É relatada, na literatura especializada, a dificuldade de se estabelecer um indicador confiável para determinar a deficiência de $\mathrm{Zn}$, pois os índices existentes atualmente apresentam limitações que dificultam o diagnóstico correto, como as dificuldades encontradas nos processos analíticos e o mecanismo homeostático do $\mathrm{Zn}$, o qual representa um fator de confundimento para a interpretação dos resultados ${ }^{(5,7,14)}$.

\section{Avaliação laboratorial dos níveis de Zn}

As dosagens de Zn plasmático e de Zn sérico são as mais utilizadas devido à facilidade da análise bioquímica em estudos epidemiológicos de grande escala. Entretanto, alguns fatores podem alterar seus resultados como, por exemplo, a inflamação, o estresse e a infecção, que diminuem a quantidade de $\mathrm{Zn}$ no plasma, indicando uma falsa deficiência de Zn. Além disso, a quantidade de Zn sérico e plasmático é afetada pela concentração de albumina, pela hemólise, pelo uso de anticoncepcionais e pelo controle homeostático ${ }^{(5,7,14,20)}$. Os valores de referência para o $\mathrm{Zn}$ sérico e plasmático foram estabelecidos pelo estudo National Health and Nutrition Examination Survey (NHANES, 1976-1980), sendo o ponto de corte de $70 \mathrm{mcg} / \mathrm{dL}$ para o plasmático e a faixa de 84 a $96 \mathrm{mcg} / \mathrm{dL}$ para o sérico, de acordo com a idade estudada ${ }^{(5)}$; entretanto, alguns autores utilizam outros pontos de corte para o Zn plasmático como $75 \mathrm{mcg} / \mathrm{dL}$ ou $80 \mathrm{mcg} / \mathrm{dL}^{(4,21,22)}$.

A análise do $\mathrm{Zn}$ eritrocitário se mostra mais elaborada do que a análise do Zn plasmático e, por isso, seu uso fica limitado a populações pequenas e médias. A meia-vida dos eritrócitos é longa (120 dias) e a quantidade de $\mathrm{Zn}$ contida neles não reflete mudanças recentes ${ }^{(5)}$. Os estudos que analisaram o $\mathrm{Zn}$ nos eritrócitos são conflitantes, encontrando níveis muito diferentes numa mesma amostra sem que tivessem correlação com a idade dos indivíduos ${ }^{(5,15,23)}$. Em uma pesquisa com pacientes portadores de doença renal crônica, o Zn plasmático foi baixo e o Zn eritrocitário foi elevado em relação ao ponto de corte utilizado pelos autores ${ }^{(24)}$. Com isso, parece que a quantidade de Zn nos eritrócitos não é constante em uma população de indivíduos semelhantes e é conflitante com os níveis de Zn plasmático, confundindo a interpretação dos resultados.

Quanto ao indicador Zn leucocitário, o primeiro estudo que utilizou os leucócitos como indicadores da quantidade de Zn corporal foi o de Vallee e Gibson, em $1948^{(25)}$ e as dificuldades encontradas pelos autores persistem até hoje. Esse índice, assim como o Zn eritrocitário, pode ser dosado apenas em grupos pequenos por ser de difícil análise, podendo ocorrer contaminação com o $\mathrm{Zn}$ presente no ambiente e na vidraria, além da possibilidade de restarem eritrócitos e plaquetas, o que superestimaria o valor do $\mathrm{Zn}$ leucocitário ${ }^{(5)}$. Mesmo com essas dificuldades, alguns estudos têm afirmado que o $Z n$ leucocitário é o biomarcador de maior confiança em comparação ao $\mathrm{Zn}$ plasmático e eritrocitário pelo fato de não variar com a idade e refletir mudanças recentes do conteúdo de Zn, já que os leucócitos possuem meia-vida mais curta do que os eritrócitos ${ }^{(26,27)}$. Para determinar o Zn leucocitário não há um consenso sobre a melhor técnica a ser utilizada e há a necessidade de grande volume sanguíneo, impossibilitando seu uso em crianças de menor idade ${ }^{(5)}$. Nessa revisão, foram observadas variações muito grandes dos níveis médios de Zn leucocitário e seu desvio padrão mesmo em indivíduos saudáveis ${ }^{(15,20,25)}$, o que pode significar que o metabolismo do Zn nos leucócitos não é constante na população saudável e, provavelmente, a sua interpretação é ainda mais difícil em pessoas doentes ${ }^{(28)}$.

Foram encontrados poucos estudos recentes que investigaram a quantidade de Zn presente nos leucócitos, já que, atualmente, existe maior interesse pela nutrigenômica, na qual os pesquisadores tentam entender a variação da quantidade de Zn nos leucócitos por meio do comportamento da expressão dos genes relacionados ao Zn e aos leucócitos. Há evidências de que a homeostase de $\mathrm{Zn}$ nos leucócitos é regulada por diferentes expressões dos genes exportadores de $\mathrm{Zn}^{(29,30)}$.

A Tabela 1 faz uma comparação dos indicadores laboratoriais do estado de $\mathrm{Zn}$ mais utilizados nos artigos revisados, confrontando-se os fatores favoráveis e limitantes de cada indicador, conforme conclusões dos autores dos referidos artigos.

\section{Outros indicadores de avaliação dos níveis de $\mathrm{Zn}$}

Outros indicadores que podem revelar o estado do Zn no organismo são avaliados por alguns pesquisadores, como a con- 
Tabela 1 - Comparação dos indicadores Zn plasmático e sérico, Zn eritrocitário e Zn leucocitário quanto aos seus fatores favoráveis e limitantes

\begin{tabular}{|c|c|c|}
\hline Indicadores & Fatores favoráveis & Fatores limitantes \\
\hline \multirow[t]{4}{*}{ Plasmático e sérico } & \multirow{4}{*}{$\begin{array}{l}\text { Facilidade da análise } \\
\text { Aplicável a grandes populações } \\
\text { Único índice com valor de referência } \\
\text { estabelecido pelo NHANES }\end{array}$} & Inflamação, estresse, infecção, hemólise \\
\hline & & e uso de anticoncepcionais alteram a \\
\hline & & quantidade de Zn no plasma e soro \\
\hline & & $\begin{array}{l}\text { Quantidade de albumina sanguínea altera a } \\
\text { concentração de Zn plasmático }\end{array}$ \\
\hline \multirow[t]{3}{*}{ Eritrocitário } & Altas concentrações de Zn nos eritrócitos & Meia-vida longa dos eritrócitos \\
\hline & Facilidade regular da análise & \multirow{2}{*}{$\begin{array}{l}\text { Em uma população formada por indivíduos } \\
\text { semelhantes, são encontrados níveis muito } \\
\text { diferentes de Zn eritrocitário }\end{array}$} \\
\hline & Aplicável em médias populações & \\
\hline \multirow[t]{4}{*}{ Leucocitário } & $\begin{array}{l}\text { Reflete mudanças recentes devido ao fato } \\
\text { de os leucócitos terem meia-vida curta }\end{array}$ & $\begin{array}{l}\text { Análise difícil pela contaminação com } \\
\text { hemácias ou plaquetas }\end{array}$ \\
\hline & \multirow{3}{*}{$\begin{array}{l}\text { Quantidade de Zn por número de glóbulos } \\
\text { brancos é maior do que a quantidade de Zn } \\
\text { por número de glóbulos vermelhos }\end{array}$} & Aplicável apenas a pequenas populações \\
\hline & & $\begin{array}{l}\text { Necessita de grande volume sanguíneo, o } \\
\text { que limita sua aplicação em lactantes }\end{array}$ \\
\hline & & $\begin{array}{l}\text { Em uma população homogênea, são } \\
\text { encontrados níveis muito diferentes de Zn } \\
\text { leucocitário }\end{array}$ \\
\hline
\end{tabular}

Os dados desta tabela são uma compilação dos resultados apresentados nos artigos revisados.

centração de Zn na urina, nas fezes, no cabelo e na saliva, mas tais estudos mostraram que não é possível usar esses fatores como índices de confiança do real estado de $\mathrm{Zn}$ no organismo ${ }^{(27,31)}$. Algumas enzimas dependentes de Zn também são estudadas como bons indicadores, principalmente a fosfatase alcalina, a desidrogenase lática e a ribonuclease; contudo, os resultados são controversos $^{(2,7,32)}$. Um maior consenso parece existir em relação à metalotioneína eritrocitária, proteína dependente de Zn que responde rapidamente a experimentos com dietas primeiramente pobres e depois ricas em $\mathrm{Zn}$, sendo diretamente proporcional à quantidade de $\mathrm{Zn}$ existente nessas dietas ${ }^{(27,33,34)}$. Recentemente, estudos genéticos vêm sendo realizados para que se encontrem os genes associados ao metabolismo do Zn e, assim, seja definido um indicador mais preciso do diagnóstico de deficiência desse mineral no organismo ${ }^{(35-40)}$.

\section{Deficiência de $\mathrm{Zn}$ em crianças com hepatopatias crônicas}

São escassos os estudos que avaliam o nível de Zn em crianças e adolescentes portadores de doenças hepáticas crônicas. Em certas doenças, como a hepatite autoimune e a deficiência de alfa-1-antitripsina, não existem estudos exclusivos, o que seria de grande interesse já que a hepatite autoimune tem pico de incidência na adolescência e a deficiência de alfa-1antitripsina é a doença de origem genética que mais causa problemas hepáticos. Existem algumas investigações que avaliam grupos de pacientes com diversas doenças hepáticas. Por exemplo, uma pesquisa de 2005 analisou a concentração de Zn plasmático em 31 crianças e adolescentes com cirrose, das quais nove tinham o diagnóstico de hepatite autoimune, mas os resultados são apresentados para o grupo total e não especificamente para os pacientes com hepatite autoimune. Os autores encontraram $42 \%$ de deficiência de Zn plasmático no grupo de pacientes. A deficiência está relacionada à gravidade da doença e à presença de colestase ${ }^{(41)}$.

Em outro estudo conduzido por Saner $e t$ al, em $2000^{(42)}$, os níveis de $\mathrm{Zn}$ foram avaliados em crianças com doenças hepáticas crônicas, subdivididas em hepatite crônica ativa e persistente. A média de Zn sérico foi significativamente menor nos pacientes do que no Grupo Controle e, quando os níveis de Zn sérico foram comparados entre os subgrupos de pacientes, observou-se que os portadores de hepatite crônica ativa apresentaram $\mathrm{Zn}$ sérico menor do aqueles com hepatite crônica persistente. O conteúdo de $\mathrm{Zn}$ nos fios de cabelos foi significativamente maior nos pacientes do que nos controles, sendo mais elevado naqueles com hepatite crônica ativa. A excreção urinária de Zn foi maior nos pa- 
cientes com hepatite crônica ativa do que nos portadores de hepatite crônica persistente. Verificou-se que a gravidade da doença hepática altera o estado de $\mathrm{Zn}$ corporal, causando a deficiência de $\mathrm{Zn}$ provavelmente por erros no mecanismo de distribuição e excreção deste nutriente. Por isso, os autores da pesquisa consideraram indispensável a suplementação de Zn em pacientes com doenças hepáticas crônicas.

$\mathrm{Na}$ hepatite B, o nível de Zn das crianças parece ser um fator preditivo para uma boa resposta à terapia com interferon. Quanto maior o nível de Zn sérico nesses pacientes, melhor a resposta a essa terapia ${ }^{(43)}$. Além disso, o nível de $Z n$ sérico em crianças com hepatite $\mathrm{B}$ foi relacionado à atividade histológica e à inflamação portal, sendo que esses pacientes não têm deficiência de $\mathrm{Zn}$, mas dependem de um elevado valor de $\mathrm{Zn}$ sérico para uma boa resposta ao tratamento da doença ${ }^{(44)}$.

$\mathrm{Na}$ hepatite $\mathrm{C}$, a suplementação com $\mathrm{Zn}$ melhorou a resposta ao tratamento com interferon e controlou o eritema acral necrolítico presente nos pacientes. Antes da suplementação, o nível de $\mathrm{Zn}$ sérico era normal nos pacientes com hepatite $\mathrm{C}$, mas ainda assim optou-se pela suplementação de Zn devido ao auxílio que este mineral fornece à resposta ao tratamento e ao controle das lesões cutâneas ${ }^{(45)}$. Assim, nos pacientes com hepatite $\mathrm{B}$ ou $\mathrm{C}$, foi verificado que, apesar de o nível de Zn sérico estar na faixa adequada, a suplementação de $Z n$ foi benéfica, principalmente para uma melhor resposta ao medicamento utilizado, podendo-se suspeitar que o ponto de corte para o Zn sérico nesses pacientes deveria ser maior do que o utilizado para indivíduos saudáveis.

Na doença de Wilson, caracterizada por erro no metabolismo de cobre $(\mathrm{Cu})$ com a ocorrência de excesso desse mineral no fígado, o enfoque quanto ao $\mathrm{Zn}$ é diferente das demais doenças hepáticas crônicas, pois a sua suplementação é uma das opções de tratamento para essa doença devido à função do Zn de competir com o cobre no processo de absorção intestinal. A alta eficácia do tratamento com $\mathrm{Zn}$ na doença de Wilson já é reconhecida na literatura e utilizada na maioria dos pacientes pediátricos ${ }^{(46,47)}$. Com isso, os valores sanguíneos de Zn não são comumente analisados nos estudos de pacientes portadores de doença de Wilson, entretanto, podese considerar que, em portadores da hepatopatia crônica não tratados, a deficiência de Zn é possível, já que o excesso de cobre provoca este fenômeno por meio da competição dos dois metais na absorção intestinal. Por isso, é muito importante tratar a doença de Wilson logo no início de suas manifestações clínicas, não só para controle da doença, mas também para evitar a deficiência de $\mathrm{Zn}$ nas crianças, impedindo futuras consequências ao seu crescimento e desenvolvimento.
Com relação à Síndrome de Alagille, foi encontrado apenas um estudo a respeito do nível de $Z n$ corporal, realizado em uma mulher adulta. Nesta paciente, o Zn sérico mostrava valor abaixo da normalidade ${ }^{(48)}$. Uma pesquisa genética que teve como objetivo identificar e mapear os genes relacionados ao Zn humano mostrou que um desses genes foi localizado em região deficiente na Síndrome de Alagille, o que indica a importância da avaliação da deficiência de $\mathrm{Zn}$ nesse grupo de pacientes ${ }^{(49)}$.

Narkewicz et al ${ }^{(50)}$ mostraram que $48 \%$ das crianças hepatopatas crônicas estudadas e que aguardam por transplante hepático apresentavam deficiência de Zn plasmático. Dentre essas crianças, a excreção urinária do mineral mostrou-se alta e a concentração de albumina, proteína ligante ao Zn plasmático, foi baixa. Após 14 dias da realização do transplante hepático, as crianças anteriormente deficientes em $\mathrm{Zn}$ apresentaram valores plasmáticos normais e redução da perda de Zn na urina. Houve correlação do nível do Zn plasmático com a albumina, mas não com a fosfatase alcalina, enzima dependente de Zn. As doenças apresentadas pelas crianças do referido estudo eram variadas, compreendendo atresia biliar, falência hepática fulminante não viral, hepatite autoimune, Síndrome de Alagille, colangite esclerosante primária, doença hepática metabólica, estreitamento do ducto biliar pós-transplante hepático, doença de Wilson e Síndrome de Budd Chiari. Infelizmente, os autores não dizem quais doenças tinham as crianças que apresentaram deficiência de $\mathrm{Zn}$ antes do transplante, mas concluem tratar-se de uma deficiência comum em crianças e adolescentes com doenças hepáticas crônicas de alta gravidade e à espera de transplante hepático. Os autores sugerem o uso de mais de um índice marcador do estado de $\mathrm{Zn}$ para interpretar melhor os dados, já que o $\mathrm{Zn}$ plasmático não é considerado um bom indicador. No caso, os autores utilizaram, além do Zn plasmático, o Zn urinário, levantando a hipótese de que o fígado prejudicado pela doença é um importante fator de desregulação da homeostase renal de Zn, a qual é corrigida após o transplante hepático.

Em outro trabalho com crianças portadoras de doença colestática hepática crônica no estágio final e à espera de transplante hepático, os autores observaram elevação do nível de $\mathrm{Zn}$ no tecido hepático, fato não compreendido ${ }^{(51)}$. Esse e outros estudos sugerem que as crianças com doenças hepáticas crônicas têm um metabolismo de $\mathrm{Zn}$ alterado. O mineral parece se concentrar no fígado e não ser corretamente distribuído aos outros tecidos do corpo, como o sangue, levando a baixos valores de $Z n$ no plasma e no soro. Entretanto, a quantidade de $Z n$ dentro das células sanguíneas ainda não foi investigada nos estudos de crianças hepatopatas crônicas. 
Em adultos com doenças hepáticas crônicas, a investigação do nível de $\mathrm{Zn}$ é mais frequente e tais estudos apresentam mais de um indicador do estado de $\mathrm{Zn}$ corporal, além da polêmica do melhor biomarcador para diagnosticar a deficiência de $\mathrm{Zn}$. Ressalta-se que o público alvo mais importante para pesquisas referentes à Zn são as crianças e adolescentes, já que, nos adultos, os sinais e sintomas da deficiência deste mineral são menos graves e intensos.

Peretz et al ${ }^{(52)}$ estudaram adultos com cirrose alcoólica e mostraram que o Zn plasmático era baixo e o Zn leucocitário, normal (frações de células mononucleadas e polimorfonucleadas). Os autores não encontraram correlação do Zn leucocitário com o Zn plasmático, com a idade dos pacientes, com o nível de albumina e nem com a fosfatase alcalina ${ }^{(28)}$. Em outra pesquisa também se encontraram níveis de Zn plasmático baixos em pacientes com cirrose alcoólica ou não alcoólica, correlacionando-se ao nível de albumina sanguínea. Entretanto, o Zn leucocitário foi baixo somente na fração de células polimorfonucleadas dos casos mais graves (presença de ascite, encefalopatia hepática, índices alterados de albumina, bilirrubina e tempo de protrombina). Nesta pesquisa, o Zn eritrocitário foi elevado nos pacientes com cirrose alcoólica, mostrando que o Zn plasmático e o leucocitário, analisados ao mesmo tempo, oferecem indicadores de confiança maior do que o Zn eritrocitário.

A mesma preocupação com os casos mais graves de cirrose hepática é relatada em outro estudo em que os pacientes cirróticos descompensados tiveram menor valor de $\mathrm{Zn}$ sérico, quando comparados aos controles e aos cirróticos compensados. Nos casos de cirrose descompensada, a excreção de Zn urinário foi maior e relacionada ao uso de diuréticos. A hiperzincúria foi considerada a principal responsável pelos baixos níveis de $\mathrm{Zn}$ sérico, juntamente com a pequena ingestão dietética de $Z n$ e de proteínas e com a má-absorção intestinal de Zn. Também nessa pesquisa os autores verificaram que a suplementação com
Zn levou à diminuição da amônia sanguínea, contribuindo para evitar a encefalopatia hepática nos pacientes com cirrose descompensada ${ }^{(53)}$. É importante lembrar que, na cirrose descompensada, há muitas vezes a orientação médica para uma dieta específica, por exemplo, com a exclusão de alimentos de origem animal a fim de diminuir a ingestão de aminoácidos que poderiam potencializar a sintomatologia clínica da doença. São justamente tais alimentos que contêm maior quantidade de $\mathrm{Zn}$, facilitando o estabelecimento de deficiência de $\mathrm{Zn}$ em pacientes mais graves.

Dentre todos os fatores que podem levar à deficiência de $\mathrm{Zn}$, ressalta-se o uso de medicamentos diuréticos e corticoides (muitas vezes administrados em pacientes hepatopatas), os quais levam à alta excreção de Zn pela urina ${ }^{(54,55)}$. Além disso, as pesquisas relatam que a deficiência de Zn plasmático é muitas vezes relacionada à quantidade de ácido fítico presente nas refeições dos indivíduos, substância antagonista à absorção de $\mathrm{Zn}^{(15)}$.

\section{Comentários e sugestões para os próximos estudos}

É de grande importância investigar o estado de Zn corporal em crianças com doenças hepáticas crônicas devido aos estudos que já comprovaram a deficiência de Zn ou a eficácia da suplementação deste mineral nesta população. Além disso, é essencial utilizar mais de um indicador para se definir a real deficiência de $\mathrm{Zn}$ no organismo, também contribuindo para estabelecer bons parâmetros que reflitam resultados confiáveis dos níveis corporais deste nutriente. É necessário relacionar os níveis sanguíneos de Zn à ingestão alimentar do mineral e aos fatores antagonistas da absorção de $\mathrm{Zn}$, tema pouco investigado nas pesquisas em crianças e adolescentes com doenças hepáticas crônicas.

\section{Referências bibliográficas}

1. Silva AP, Vitolo MR, Zara LF, Castro CF. Effects of zinc supplementation on 1-to-5-year-old children. J Pediatr (Rio J) 2006;82:227-31.

2. Mafra D, Cozzolino SM. The importance of zinc in human nutrition. Rev Nutr 2004;17:79-87.

3. Marques RC, Marreiro DN. Metabolic and functional aspects of zinc in Down syndrome. Rev Nutr 2006;19:501-10.

4. Yorbik O, Ozdag MF, Olgun A, Senol MG, Bek S, Akman S. Potential effects of zinc on information processing in boys with attention deficit hyperactivity disorder. Prog Neuropsychopharmacol Biol Psychiatry 2008;32:662-7.

5. Gibson RS. Assessment of trace-element status. In: Gibson RS, editor. Principles of nutritional assessment. New York: Oxford University Press; 1990. p. 511-53.
6. Mocchegiani E, Muzzioli M, Giacconi R. Zinc and immunoresistance to infection in aging: new biological tools. Trends Pharmacol Sci 2000;21:205-8.

7. Wood RJ.Assessmentof marginal zincstatus in humans. J Nutr 2000;130 (Suppl5): 1350-4S.

8. Food and Nutrition Board, Institute of Medicine. Zinc. In: National Academy of Sciences, editor. Dietary reference intakes for vitamin A, vitamin k, arsenic, boron, chromium, copper, iodine, iron, manganese, molybdenum, nickel, silicon, vanadium, and zinc. Washington: National Academy Press; 2000. p. 442-501.

9. Aggett PJ, Comerford JG. Zinc in human health. Nutr Rev 1995;53:16-22S.

10. Lönnerdal B. Dietary factors influencing zinc absorption. J Nutr 2000;130 (Suppl5): 1378-83S. 
11. Jackson MJ. Physiology of zinc: general aspects. In: Mills CF, editor. Zinc in human biology. London: Springer-Verlag; 1989. p. 323-33.

12. Hinks LJ, Clayton BE. Zinc and copper concentrations in leucocytes and eritrocytes in healthy adults and the effect of oral contraceptives. J Clin Pathol 1983;36:1016-21.

13. Foote JW, Delves HT. Distribution of zinc amongst human serum proteins determined by affinity chromatography and atomic-absorption spectrophotometry. Analyst 1983;108:492-504.

14. No authors listed. Zinc and health: current status and future directions. J Nutr 2000;130 (Suppl 5):1341-3S.

15. Hambidge M. Underwood Memorial Lecture: human zinc homeostasis: good but not perfect. J Nutr 2003;133 (Suppl 1):1438-42S.

16. Krebs NF, Hambidge M, Westcott JE, Miller LV, Sian L, Bell M et al. Exchangeable zinc pool size in infants is related to key variables of zinc homeostasis. J Nutr 2003;133 (Suppl 1):1498-501S.

17. Prasad AS, Miale A Jr, Farid Z, Sandstead HH, Schulert AR. Zinc metabolism in patients with the syndrome of iron deficiency anemia, hepatosplenomegaly, dwarfism, and hypognadism. J Lab Clin Med 1963;61:537-49.

18. Prasad AS. Zinc deficiency in women, infants and children. J Am Coll Nutr 1996;15:113-20.

19. MacDonald RS. The role of zinc in growth and cell proliferation. J Nutr 2000;130 (Suppl 5):1500-8S

20. Hinks LJ, Colmsee M, Delves HT. Determination of zinc and copper in isolated leucocytes. Analyst 1982;107:815-23.

21. Sheng XY, Hambidge KM, Zhu XX, Ni JX, Bailey KB, Gibson RS et al. Major variables of zinc homeostasis in Chinese toddlers. Am J Clin Nutr 2006;84: 389-94.

22. Mafra D, Cuppari L, Fávaro DI, Cozzolino SM. Zinc levels after iron supplementation in patients with chronic kidney disease. J Ren Nutr 2004;14:164-9.

23. Nishi Y. Zinc levels in plasma, erythrocyte and leukocyte in healthy children and adults. Hiroshima J Med Sci 1980;29:7-13.

24. Mafra D, Cozzolino SM. Erythrocyte zinc and carbonic anhydrase levels in nondialyzed chronic kidney disease patients. Clin Biochem 2004;37: 67-71.

25. Vallee BL, Gibson JG. The zinc content of normal human whole blood, plasma, leucocytes, and erythrocytes. J Biol Chem 1948;176:445-57.

26. Caticha O, Norato DY, Tambascia MA, Santana A, Stephanou A, Sarlis NJ. Total body zinc depletion and its relationship to the development of hyperprolactinemia in chronic renal insufficiency. J Endocrinol Invest 1996;19:441-8.

27. Hambidge M. Biomarkers of trace mineral intake and status. J Nutr 2003; 133 (Suppl 3):948S-55S.

28. Peretz A, Nève J, Jeghers O, Leclercq N, Praet JP, Vertongen F et al. Interest of zinc determination in leucocyte fractions for the assessment of marginal zinc status. Clin Chim Acta 1991;203:35-46.

29. Overbeck S, Uciechowski P, Ackland ML, Ford D, Rink L. Intracellular zinc homeostasis in leukocyte subsets is regulated by different expression of zinc exporters ZnT-1 to ZnT-9. J Leukoc Biol 2008;83:368-80.

30. Haase H, Mazzatti DJ, White A, Ibs KH, Engelhardt G, Hebel S et al. Differential gene expression after zinc supplementation and deprivation in human leukocyte subsets. Mol Med 2007;13:362-70.

31. Kanabrocki EL, Ryan MD, Marks G, Friedman NC, Kaplan E, Nemchausky BA. Twenty-nine year study on circadian distribution of urinary zinc levels of same male subjects. Clin Ter 2007;158:403-8.

32. McCall KA, Huang $C$, Fierke CA. Function and mechanism of zinc metalloenzymes. J Nutr 2000;130 (Suppl 5):1437-46S.

33. Sullivan VK, Burnett FR, Cousins RJ. Metallothionein expression is increased in monocytes and erythrocytes of young men during zinc supplementation. $J$ Nutr 1998;128:707-13.

34. Thomas EA, Bailey LB, Kauwell GA, Lee DY, Cousins RJ. Erythrocyte metallothionein response to dietary zinc in humans. J Nutr 1992;122: 2408-14.
35. Mazzatti DJ, Malavolta M, White AJ, Costarelli L, Giacconi R, Muti E et al. Differential effects of in vitro zinc treatment on gene expression in peripheral blood mononuclear cells derived from young and elderly individuals. Rejuvenation Res 2007;10:603-20.

36. Cousins RJ, McMahon RJ. Integrative aspects of zinc transporters. J Nutr 2000;130 (Suppl 5):1384-7S.

37. Dieck HT, Döring F, Roth HP, Daniel H. Changes in rat hepatic gene expression in response to zinc deficiency as assessed by DNA arrays. J Nutr 2003;133:1004-10.

38. Cui L, Blanchard RK, Cousins RJ. The permissive effect of zinc deficiency on uroguanylin and inducible nitric oxide synthase gene upregulation in rat intestine induced by interleukin $1 \alpha$ is rapidly reversed by zinc repletion. J Nutr 2003;133:51-6.

39. Cousins RJ, Blanchard RK, Moore JB, Cui L, Green CL, Liuzzi JP et al. Regulation of zinc metabolism and genomic outcomes. J Nutr 2003;133 (Suppl 1): 1521-6S.

40. Cousins RJ, Liuzzi JP, Lichten LA. Mammalian zinc transport, trafficking, and signals. J Biol Chem 2006;281:24085-9.

41. Schneider ACR, Pinto RB, Froehlich PE, Borges AP, Maldonado A, da Silveira TR. Zinco plasmático em crianças e adolescentes com cirrose. Resumo do XII Congresso Brasileiro de Gastroenterologia Pediátrica e II Congresso Brasileiro de Hepatologia Pediátrica; 2005 Nov 5-9; Gramado, Brasil. p. 16.

42. Saner G, Suoglu OD, Yigitbasi M, Sokucu S, Elkabes B. Zinc nutrition in children with chronic liver disease. J Trace Elem Exp Med 2000;13:271-6.

43. Ozbal E, Helvaci M, Kasirga E, Akdenizoglu F, Kizilgünesler A. Serum zinc as a factor predicting response to interferon-alpha2b therapy in children with chronic hepatitis B. Biol Trace Elem Res 2002;90:31-8.

44. Selimoglu MA, Aydogdu S, Unal F, Yüce G, Yagci RV. Serum zinc status in chronic hepatitis $B$ and its relationship to liver histology and treatment results. Pediatr Int 2001;43:396-9.

45. El-Ghandour TM, Sakr MA, El-Sebai H, El-Gammal TF, El-Sayed MH. Necrolytic acral erythema in Egyptian patients with hepatitis $C$ virus infection. J Gastroenterol Hepatol 2006;21:1200-6.

46. Hoogenraad TU. Paradigm shift in treatment of Wilson's disease: zinc therapy now treatment of choice. Brain Dev 2006;28:141-6.

47. Merle U, Schaefer M, Ferenci P, Stremmel W. Clinical presentation, diagnosis and long-term outcome of Wilson's disease: a cohort study. Gut 2007;56: 115-20.

48. Hofbauer LC, Mrozek-Lasota A, Jelinek T, Schworm HD, Zimmermann $D$, Heufelder AE. Endocrinologic and metabolic complications of Alagille syndrome. Med Klin (Munich) 1997;92:528-33.

49. Tommerup $\mathrm{N}$, Vissing $\mathrm{H}$. Isolation and fine mapping of 16 novel human zinc finger-encoding cDNAs identify putative candidate genes for developmental and malignant disorders. Genomics 1995;27:259-64.

50. Narkewicz MR, Krebs N, Karrer F, Orban-Eller K, Sokol RJ. Correction of hypozincemia following liver transplantation in children is associated with reduced urinary zinc loss. Hepatology 1999;29:830-3.

51. Phillips MJ, Ackerley CA, Superina RA, Roberts EA, Filler RM, Levy GA. Excess zinc associated with severe progressive cholestasis in Cree and Ojibwa-Cree children. Lancet 1996;347:866-8.

52. Goode HF, Kelleher J, Walker BE. Relation between zinc status and hepatic functional reserve in patients with liver disease. Gut 1990;31:694-7.

53. Yoshida Y, Higashi T, Nouso K, Nakatsukasa H, Nakamura SI, Watanabe A et al. Effects of zinc deficiency/zinc supplementation on ammonia metabolism in patients with decompensated liver cirrhosis. Acta Med Okayama 2001;55:349-55.

54. Peretz A, Neve J, Famaey JP. Effects of chronic and acute corticosteroid therapy on zinc and copper status in rheumatoid arthritis patients. J Trace Elem Electrolytes Health Dis 1989;3:103-8.

55. Milanino R, Frigo A, Bambara LM, Marrella M, Moretti U, Pasqualicchio $\mathrm{M}$ et al. Copper and zinc status in rheumatoid arthritis: studies of plasma, erythrocytes, and urine, and their relationship to disease activity markers and pharmacological treatment. Clin Exp Rheumatol 1993;11:271-81. 